\title{
Test and Commissioning of the AST3-1 Control System
}

\author{
Xiaoyan $\mathbf{L i}^{1,2,3}$ and Daxing Wang ${ }^{1,3}$ \\ ${ }^{1}$ National Astronomical Observatories/Nanjing Institute of Astronomical \\ Optics \& Technology, Chinese Academy of Sciences, \\ Nanjing 210042, China \\ ${ }^{2}$ Email: xyli@niaot.ac.cn \\ ${ }^{3}$ Key Laboratory of Astronomical Optics \& Technology, Nanjing Institute of Astronomical \\ Optics \& Technology, Chinese Academy of Sciences, \\ Nanjing 210042, China
}

\begin{abstract}
The first of three Antarctic Survey Telescopes (AST3-1), a 50/68 cm Schmidt-like equatorial-mount telescope, is the first trackable Chinese telescope operating on the Antarctic plateau. It was installed at Dome A $\left(80^{\circ} 22^{\prime} \mathrm{S}, 77^{\circ} 21^{\prime} \mathrm{E}, 4,093 \mathrm{~m}\right)$, the highest place on the Antarctic plateau, in 2012. The telescope is unmanned during night-time operations through the Austral winter. The telescope optics and mechanics, as well as the motors and position sensors, are exposed to a harsh environment. The mechanics is enclosed with a foldable tent-like dome to prevent snow, diamond dust and ice. While the control cabinet containing drive boxes, circuit board boxes, power converters and the Telescope Control Computer (TCC) is located inside the warm instrumental module. In about 15 weeks remote testing and commissioning, from January 24 when the expedition team left there to May 8, when the communication failed, we obtained images with the best FWHM of less than $2^{\prime \prime}$. We also recorded the telescope movement performance and fine-tuned the dynamic properties of the telescope control system. Some experiences and lessons will be disscussed in this paper.
\end{abstract}

Keywords. AST3-1, Dome A, control system, movement performance

\section{Introduction}

The telescope control system of AST3-1, including the telescope movement system, focusing system and lens-heating system, consists of Telescope Control Software(TCS), Telescope Control Computer (TCC), driveboxes, motors, position sensors, current sensors and voltage sensors, etc. It has such features as:

Drive system: Gear drive with dual-motors per axis and drive boxes equipped with an anti-backlash control unit.

Motors: Customized AC servo PMSM (Permanent Magnet Synchronous Motor) with a two-pole resolver as the feedback sensor. Four such motors were mounted on the two axes of the telescope. Besides, a customized stepper motor was used in the focusing system. All these motors were not heated and they all have worked through lower than $-70^{\circ} \mathrm{C}$. Resolvers: Multi-pole resolvers instead of commonly used photoelectric encoders were

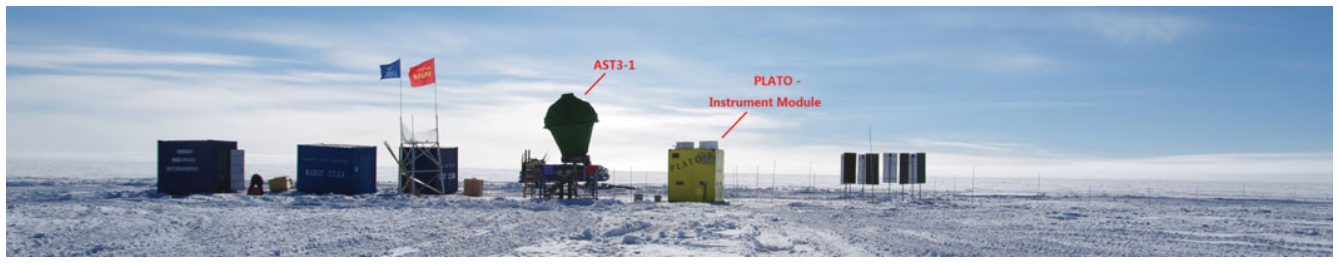



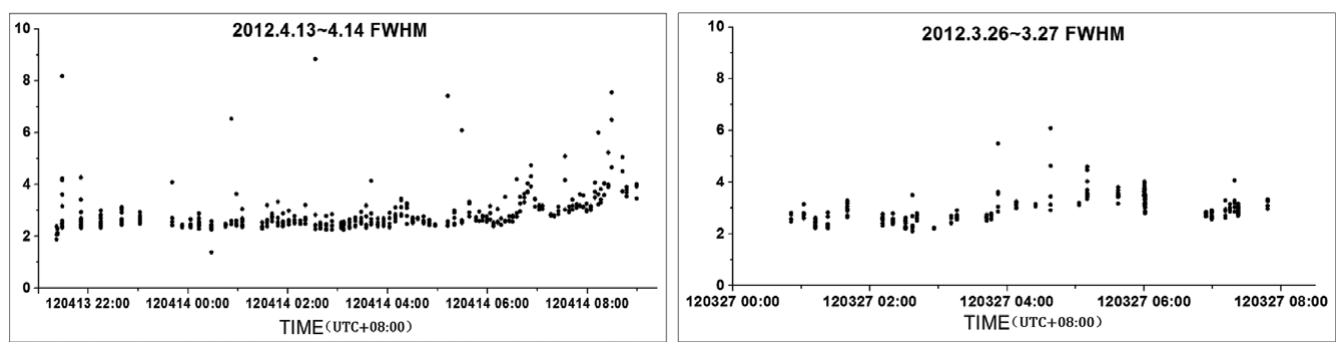

Figure 1. Average star FWHM from AST3-1 (one point stands for one image).
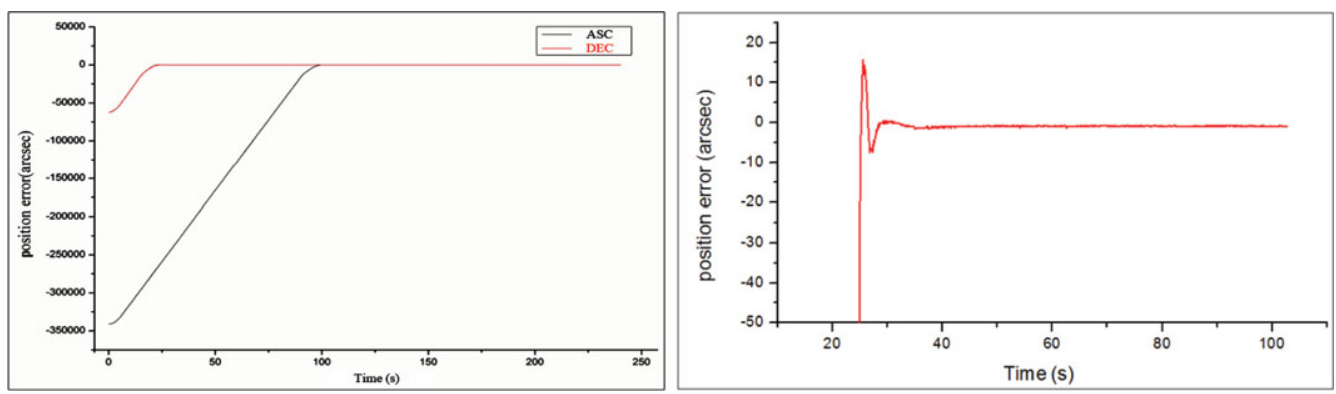

Figure 2. Asc/Dec position error.

used to obtain the position of the two telescope axes. Resolvers were not heated and they all worked well through the testing and commissioning stage.

Commands: About 50 commands provide comprehensive control and feedback of the telescope control system. The TCS, running on the TCC, receives and interprets these commands and manages the control system.

Protection: Various auxilliary and safety sensors help protect the telescope to keep it running safely.

\section{Test and Commissioning Result}

The control system of AST3-1 has worked through ambient temperatures lower than $-70^{\circ} \mathrm{C}$. The telescope continued running until the communication dropped on May 8 . We have obtained many images in about 8-weeks of observation. Figure 1 shows the average star FWHM (Full Width at Half Maximum) of all images during one night of observing. Most of the FWHMs are between $2^{\prime \prime}$ and $3^{\prime \prime}$ demonstrate good seeing and tracking performance.

TCS recorded a quantitative measurement of the telescope movement. Accordingly, we can change the parameters of the hardware servo and software servo to fine tune the dynamic properties of the telescope. The left image of Figure 2 shows an example record of the Asc/Dec position error. When the position error get close to zero it means the telescope switches from pointing to tracking. Zoom into the intersection of pointing and tracking to obtain the right image in Figure 2. This shows a small overshoot of less than $1 \%$ and a fast transition of only one adjustment. The tracking following error of the Asc axis is less than $0.4^{\prime \prime}$ and for the Dec axis less than $0.6^{\prime \prime}$.

We also have obtained some bad images during observing on several nights. Some of these demonstrate that the weather conditions are poor at times. When the wind speed is high, the tent-like dome and the tube stiffness will affect the image quality. 